IJMMS 27:12 (2001) 765-768

PII. S0161171201006834

http://ijmms.hindawi.com

(c) Hindawi Publishing Corp.

\title{
ON THE EXTENDED HARDY'S INEQUALITY
}

\section{YAN PING}

(Received 16 February 2000)

ABSTRACT. We generalize a strengthened version of Hardy's inequality and give a new simpler proof.

2000 Mathematics Subject Classification. 26D15, 40A25.

In the recent paper [4], Hardy's inequality was generalized. In this note, the results given in [4] are further generalized and a new much simpler proof is given. The following Hardy's inequality is well known [1, Theorem 349].

THEOREM 1 (Hardy's inequality). Let $\lambda_{n}>0, A_{n}=\sum_{k=1}^{n} \lambda_{k}, a_{n} \geq 0(n \in \mathbb{N})$, $0<\sum_{n=1}^{\infty} \lambda_{n} a_{n}<+\infty$, then

$$
\sum_{n=1}^{\infty} \lambda_{n}\left(a_{1}^{\lambda_{1}} a_{2}^{\lambda_{2}} \cdots a_{n}^{\lambda_{n}}\right)^{1 / A_{n}}<e \sum_{n=1}^{\infty} \lambda_{n} a_{n}
$$

Recently, [4] gave an improvement of Theorem 1, and the following result was proved.

THEOREM 2. Let $0<\lambda_{n+1} \leq \lambda_{n}, A_{n}=\sum_{k=1}^{n} \lambda_{k}, a_{n} \geq 0(n \in \mathbb{N}), 0<\sum_{n=1}^{\infty} \lambda_{n} a_{n}<+\infty$, then

$$
\sum_{n=1}^{\infty} \lambda_{n+1}\left(a_{1}^{\lambda_{1}} a_{2}^{\lambda_{2}} \cdots a_{n}^{\lambda_{n}}\right)^{1 / A_{n}}<e \sum_{n=1}^{\infty}\left(1-\frac{\lambda_{n}}{2\left(A_{n}+\lambda_{n}\right)}\right) \lambda_{n} a_{n} .
$$

In this note, we will prove the following theorem.

THEOREM 3. Let $0<\lambda_{n+1} \leq \lambda_{n}, A_{n}=\sum_{k=1}^{n} \lambda_{k}, a_{n} \geq 0(n \in \mathbb{N}), 0<\sum_{n=1}^{\infty} \lambda_{n} a_{n}<$ $+\infty$, then

$$
\sum_{n=1}^{\infty} \lambda_{n+1}\left(a_{1}^{\lambda_{1}} a_{2}^{\lambda_{2}} \cdots a_{n}^{\lambda_{n}}\right)^{1 / A_{n}}<e \sum_{n=1}^{\infty}\left(1+\frac{5 \lambda_{n}}{5 A_{n}+\lambda_{n}}\right)^{-1 / 2} \lambda_{n} a_{n} .
$$

To prove Theorem 3, we introduce some lemmas.

LEMMA 4. For $x>0$, then

$$
e\left(1-\frac{1}{2 x+1}\right)<\left(1+\frac{1}{x}\right)^{x}<e\left(1+\frac{5}{5 x+1}\right)^{-1 / 2} .
$$


Proof. (i) Define $f(x)$ as

$$
f(x)=x \ln \left(1+\frac{1}{x}\right)+\frac{1}{2} \ln \left(1+\frac{5}{5 x+1}\right), \quad x \in(0,+\infty) .
$$

It is obvious that when $x>0$, the inequality

$$
\left(1+\frac{1}{x}\right)^{x}<e\left(1+\frac{5}{5 x+1}\right)^{-1 / 2}
$$

is equivalent to $f(x)<1$. It is easy to see that

$$
f^{\prime}(x)=-\frac{1}{x+1}+\ln \left(1+\frac{1}{x}\right)-\frac{25}{2(5 x+6)(5 x+1)}
$$

and for $x \in(0,+\infty)$, it can be shown that

$$
\begin{aligned}
f^{\prime \prime}(x) & =\frac{1}{(x+1)^{2}}-\frac{1}{x(x+1)}+\frac{25}{2(5 x+1)^{2}}-\frac{25}{2(5 x+6)^{2}} \\
& =\frac{-125 x^{3}-50 x^{2}+35 x-72}{2 x(x+1)^{2}(5 x+1)^{2}(5 x+6)^{2}}<0 .
\end{aligned}
$$

Hence $f^{\prime}(x)$ is decreasing on $(0,+\infty)$. Then for any $x \in(0,+\infty)$, we have $f^{\prime}(x)>$ $\lim _{x \rightarrow+\infty} f^{\prime}(x)=0$, thus, $f(x)$ is increasing on $(0,+\infty)$, and $f(x)<\lim _{x \rightarrow+\infty} f(x)=1$ for $x \in(0,+\infty)$. The inequality (6) is valid.

(ii) Define $g(x)$ as

$$
g(x)=x \ln \left(1+\frac{1}{x}\right)-\ln \left(1-\frac{1}{2 x+1}\right), \quad x \in(0,+\infty)
$$

When $x>0$, the inequality

$$
e\left(1-\frac{1}{2 x+1}\right)<\left(1+\frac{1}{x}\right)^{x}
$$

is equivalent to $g(x)>1$. For $x \in(0,+\infty)$, it can be shown that

$$
\begin{aligned}
& g^{\prime}(x)=-\frac{1}{x+1}+\ln \left(1+\frac{1}{x}\right)-\frac{1}{x(2 x+1)}, \\
& g^{\prime \prime}(x)=\frac{5 x^{2}+5 x+1}{x^{2}(x+1)^{2}(2 x+1)^{2}}>0 .
\end{aligned}
$$

Hence, $g^{\prime}(x)$ is increasing on $(0,+\infty)$. Then for any $x \in(0,+\infty)$, we have $g^{\prime}(x)<$ $\lim _{x \rightarrow+\infty} g^{\prime}(x)=0$, therefore, $g(x)$ is decreasing on $(0,+\infty)$ and $g(x)>\lim _{x \rightarrow+\infty} g(x)$ $=1$ for $x \in(0,+\infty)$. Inequality (10) is valid.

By virtue of (6) and (10), inequalities (4) are valid. This proves Lemma 4.

REMARK 5. By a direct calculation, we have

$$
\left(1+\frac{5}{5 x+1}\right)^{-1 / 2}<1-\frac{1}{2(x+19 / 20)} \quad(x>0) .
$$


Then by (4) and (12), we have

$$
e\left(1-\frac{1}{2 x+1}\right)<\left(1+\frac{1}{x}\right)^{x}<e\left[1-\frac{1}{2(x+19 / 20)}\right] \quad(x>0) .
$$

Inequality (13) is equivalent to

$$
\frac{e}{2(x+19 / 20)}<e-\left(1+\frac{1}{x}\right)^{x}<\frac{e}{2 x+1} \quad(x>0) .
$$

Thus, [1, Lemma 2] is contained in Lemma 4. Inequalities (4) and (14) are the new inequalities on the constant $e$ (cf. [3, Theorem 3.8.26]; and [2, page 358]).

LEMMA 6 (see [1, Theorem 9]). Let $g_{m}>0, \alpha_{m} \geq 0(m=1,2, \ldots, n), \sum_{m=1}^{n} g_{m}=1$, then

$$
\alpha_{1}^{g_{1}} \alpha_{2}^{g_{2}} \cdots \alpha_{n}^{g_{n}} \leq \sum_{m=1}^{n} g_{m} \alpha_{m}
$$

Proof of Theorem 3. Setting $c_{m}>0, g_{m}=\lambda_{m} / A_{n}, \alpha_{m}=c_{m} a_{m}(m=1,2, \ldots, n)$, by Lemma 6 , we have

$$
\left(c_{1} a_{1}\right)^{\lambda_{1} / A_{1}}\left(c_{2} a_{2}\right)^{\lambda_{2} / A_{2}} \cdots\left(c_{n} a_{n}\right)^{\lambda_{n} / A_{n}} \leq \frac{1}{A_{n}} \sum_{m=1}^{n} \lambda_{m} c_{m} a_{m} .
$$

Then we find that

$$
\begin{aligned}
\sum_{n=1}^{\infty} \lambda_{n+1}\left(a_{1}^{\lambda_{1}} a_{2}^{\lambda_{2}} \cdots a_{n}^{\lambda_{n}}\right)^{1 / A_{n}} & =\sum_{n=1}^{\infty} \lambda_{n+1} \frac{\left(c_{1} a_{1}\right)^{\lambda_{1} / A_{1}}\left(c_{2} a_{2}\right)^{\lambda_{2} / A_{2}} \cdots\left(c_{n} a_{n}\right)^{\lambda_{n} / A_{n}}}{\left(c_{1}^{\lambda_{1}} c_{2}^{\lambda_{2}} \cdots c_{n}^{\lambda_{n}}\right)^{1 / A_{n}}} \\
& \leq \sum_{n=1}^{\infty}\left[\frac{\lambda_{n+1}}{\left(c_{1}^{\lambda_{1}} c_{2}^{\lambda_{2}} \cdots c_{n}^{\lambda_{n}}\right)^{1 / A_{n}}}\right] \frac{1}{A_{n}} \sum_{m=1}^{n} c_{m} \lambda_{m} a_{m} \\
& =\sum_{m=1}^{\infty} \lambda_{m} a_{m} c_{m} \sum_{n=m}^{\infty} \frac{\lambda_{n+1}}{A_{n}\left(c_{1}^{\lambda_{1}} c_{2}^{\lambda_{2}} \cdots c_{n}^{\lambda_{n}}\right)^{1 / A_{n}}} .
\end{aligned}
$$

Define $c_{m}=\left(\left(A_{m+1}\right) / A_{m}\right)^{A_{m} / \lambda_{m}} A_{m}(m=1,2, \ldots)$ and $A_{0}=0$. Because $0<\lambda_{n+1} \leq$ $\lambda_{n}(n=1,2, \ldots)$, we have

$$
\begin{aligned}
& c_{m}^{\lambda_{m}}=\frac{\left(A_{m+1}\right)^{A_{m}}}{A_{m}^{A_{m}-1}} ; \quad\left(c_{1}^{\lambda_{1}} c_{2}^{\lambda_{2}} \cdots c_{n}^{\lambda_{n}}\right)^{1 / A_{n}}=A_{n+1} \quad(n \in \mathbb{N}) ; \\
& c_{m} \sum_{n=m}^{\infty} \frac{\lambda_{n+1}}{A_{n}\left(c_{1}^{\lambda_{1}} c_{2}^{\lambda_{2}} \cdots c_{n}^{\lambda_{n}}\right)^{1 / A_{n}}}=\left(\frac{A_{m+1}}{A_{m}}\right)^{A_{m} / \lambda_{m}} A_{m} \sum_{n=m}^{\infty} \frac{\lambda_{n+1}}{A_{n} A_{n+1}} \\
&=\left(1+\frac{\lambda_{m+1}}{A_{m}}\right)^{A_{m} / \lambda_{m}} A_{m} \sum_{n=m}^{\infty}\left(\frac{1}{A_{n}}-\frac{1}{A_{n+1}}\right) \\
& \leq\left(1+\frac{\lambda_{m}}{A_{m}}\right)^{A_{m} / \lambda_{m}} .
\end{aligned}
$$


Then by (4) and (17), we obtain that

$$
\begin{aligned}
\sum_{n=1}^{\infty} \lambda_{n+1}\left(a_{1}^{\lambda_{1}} a_{2}^{\lambda_{2}} \cdots a_{n}^{\lambda_{n}}\right)^{1 / A_{n}} & \leq \sum_{m=1}^{\infty}\left(1+\frac{\lambda_{m}}{A_{m}}\right)^{A_{m} / \lambda_{m}} \lambda_{m} a_{m} \\
& \leq e \sum_{m=1}^{\infty}\left(1+\frac{5 \lambda_{m}}{5 A_{m}+\lambda_{m}}\right)^{-1 / 2} \lambda_{m} a_{m} .
\end{aligned}
$$

Hence inequality (3) is valid, and Theorem 3 is proved.

REMARK 7. With inequality (12), Theorem 3 is obviously an improvement and an extension of [4, Theorem 1].

Setting $\lambda_{n} \equiv 1$, (3) changes into

$$
\sum_{n=1}^{\infty}\left(a_{1} a_{2} \cdots a_{n}\right)^{1 / n}<e \sum_{n=1}^{\infty}\left(1+\frac{5}{5 n+1}\right)^{-1 / 2} a_{n} .
$$

By inequality (12), we have

$$
\sum_{n=1}^{\infty}\left(a_{1} a_{2} \cdots a_{n}\right)^{1 / n}<e \sum_{n=1}^{\infty}\left[1-\frac{1}{2(n+19 / 20)}\right] a_{n} .
$$

Thus, inequalities (20) and (21) are obviously an improvement and extension of [5, Theorem 3.1].

\section{REFERENCES}

[1] G. H. Hardy, J. E. Littlewood, and G. Pólya, Inequalities, 2nd ed., Cambridge University Press, Cambridge, 1952. MR 13,727e. Zbl 047.05302.

[2] J. C. Kuang, Changyong budengshi [Applied Inequalities], 2nd ed., Hunan Jiaoyu Chubanshe, Changsha, 1993 (Chinese). MR 95j:26001.

[3] D. S. Mitrinović, Analytic Inequalities, Die Grundlehren der mathematischen Wisenschaften, vol. 165, Springer-Verlag, New York, 1970. MR 43\#448. Zbl 0199.38101.

[4] B. Yang, On Hardy's inequality, J. Math. Anal. Appl. 234 (1999), no. 2, 717-722. MR 2000b:26032. Zbl 946.26011.

[5] B. Yang and L. Debnath, Some inequalities involving the constant e, and an application to Carleman's inequality, J. Math. Anal. Appl. 223 (1998), no. 1, 347-353. MR 99h:26026. Zbl 910.26011.

YAN Ping: DePaRtment of Mathematics, ANHUi Normal University, WuHu City, ANHUi 241000, CHINA

Current address: DePARTMENT OF MATHEMATICS, University OF TURKU, FIN-20014 TURKU, FINLAND 


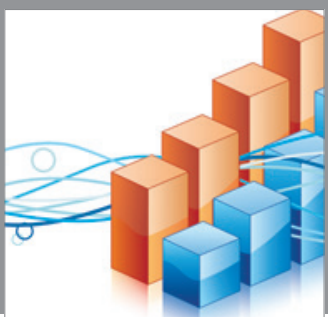

Advances in

Operations Research

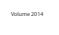

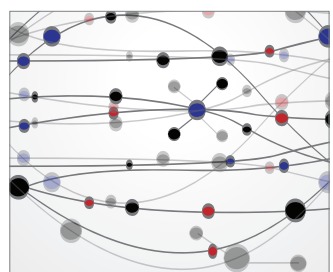

\section{The Scientific} World Journal
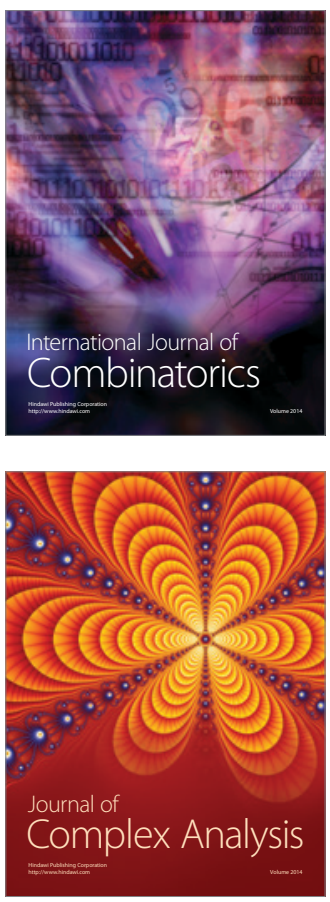

International Journal of

Mathematics and

Mathematical

Sciences
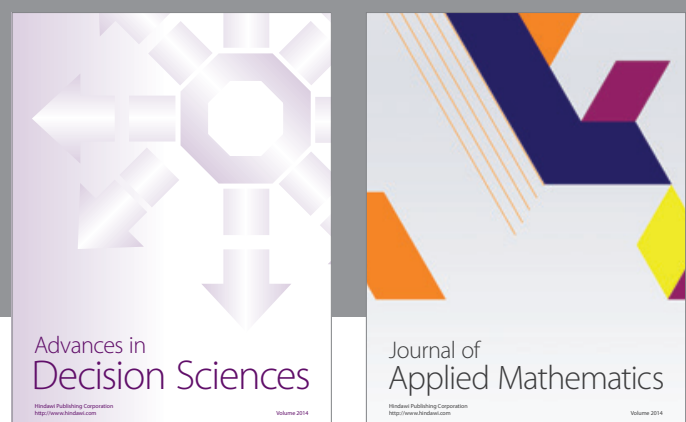

Journal of

Applied Mathematics
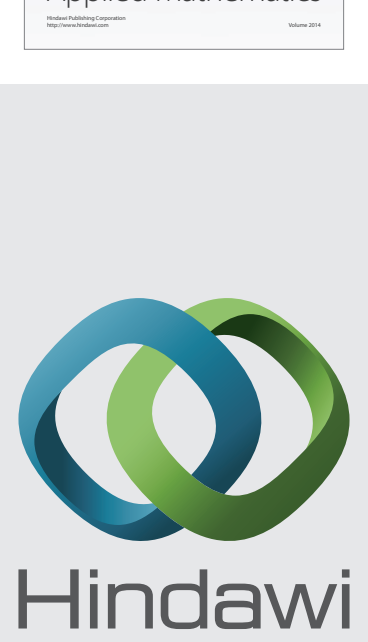

Submit your manuscripts at http://www.hindawi.com
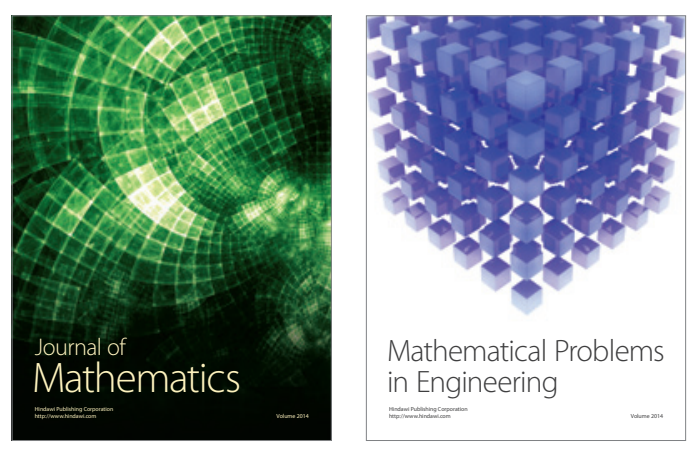

Mathematical Problems in Engineering
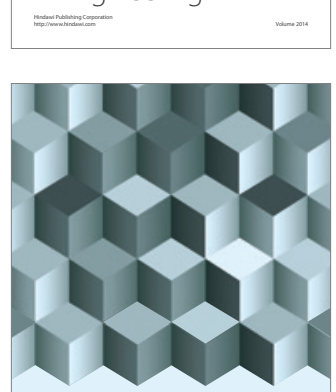

Journal of

Function Spaces
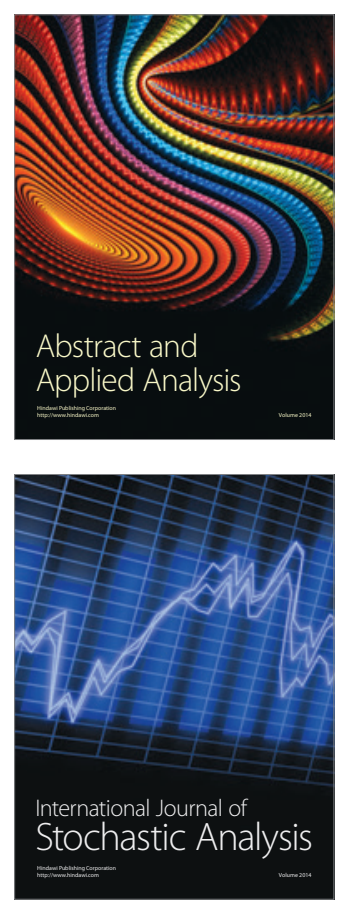

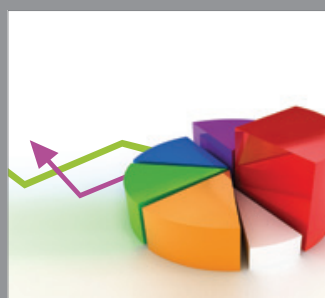

ournal of

Probability and Statistics

Promensencen
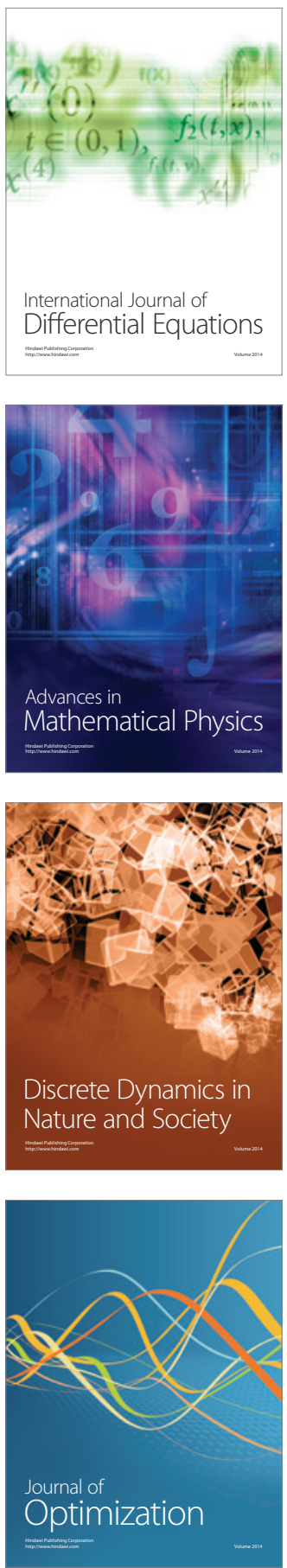